\title{
UNA SALIDA AL DUALISMO: \\ JUEGO Y TRABAJO EN JOHN DEWEY
}

\author{
AN EXIT TO DUALISM: \\ PLAY AND WORK IN JOHN DEWEY
}

\author{
Carlo RODRÍGUEz SABARIZ* \\ UNED
}

Resumen: En nuestra cultura, el juego y el trabajo suelen verse como dos esferas de acción antitéticas y sustancialmente heterogéneas. Sin embargo, John Dewey juzgó que esta visión dominante constituía uno más de los falsos dualismos en los que estamos inmersos. Dejando a un lado las convenciones culturales, Dewey analizará el juego y el trabajo como actitudes mentales que, en dialéctica con las condiciones externas, determinan el grado de satisfacción y productividad de nuestras actividades. Nuestro artículo se propone examinar estas ideas filosóficas, para a continuación, mostrar cómo Dewey se sirvió de ellas (entre otras ideas) para elaborar su propuesta pedagógica (materializada en el plan de estudios de la conocida como Laboratory School), y para construir su crítica al carácter del trabajo en las nuevas condiciones del capitalismo industrial.

Palabras Clave: dualismo, juego, trabajo, ocupaciones activas, industrialización.

АвsтRACт: In our culture, play and work are usually seen as two antithetical and substantially heterogeneous spheres of action. However, John Dewey judged this dominant view to be one more of the false dualisms in which we are immersed. Leaving aside

* Como investigador de la UNED, he elaborado este artículo dentro del Programa de Doctorado en Filosofía de la Escuela de Doctorado Internacional de la UNED. Forma parte, por lo tanto, de las actividades dirigidas por mi director de tesis doctoral, D. Ramón del Castillo Santos, como complemento a la investigación que realizo en dicho Programa acerca de un aspecto del pensamiento del filósofo John Dewey. El título de la tesis es: «Trabajo y juego en la sociedad industrial: Una perspectiva deweyana». 
the culture conventions, Dewey analyzes play and work as mental attitudes that, in a dialectic with external conditions, determine the degree of satisfaction and productivity of our activities. Our paper aims to examine these philosophical ideas, and then to show how Dewey employed them (among other ideas) to develop his pedagogical proposal (embodied in the Laboratory School' curriculum), and to construct his criticism to the character of labor in the new conditions of industrial capitalism.

KeYwORDs: dualism, play, work, active occupations, industrialization.

\section{Introducción}

El propósito de este artículo es llamar la atención sobre las ideas filosóficas de John Dewey (1859-1952) acerca del juego y el trabajo. Se trata de una cuestión que ha pasado desapercibida entre sus estudiosos e intérpretes y que, a nuestro juicio, a pesar de esta ausencia y del hecho de que Dewey nunca la abordó de forma sistemática, desempeña un importante papel en su pensamiento. Aunque mucha gente conoce a Dewey por su labor pedagógica y tiende a identificarlo como pedagogo, lo cierto es que él siempre se reconoció primeramente como filósofo. En el propio ámbito educativo, se dedicó mucho más a reflexionar sobre el lugar que la educación debía cumplir en el orden social que acerca de técnicas concretas de enseñanza y aprendizaje. Para Dewey, la filosofía no consistía más que en trabajar con ideas abstractas, y este fue su principal campo de estudio. No obstante, como buen pragmatista, no entendió las ideas abstractas como meros malabarismos intelectuales al modo del idealismo, sino como herramientas que pudieran guiar nuestra conducta y ser aplicadas en otros campos de experiencia. Como veremos, Dewey aplicó sus ideas filosóficas (y psicológicas) sobre el jugar y el trabajar en dos campos: el educativo, en particular, a la hora de diseñar el plan de estudios de la conocida como Laboratory School, la escuela de primaria que dirigió en Chicago entre 1896 y 1904; y el político-social, con su crítica al carácter del trabajo en las nuevas condiciones del capitalismo industrial y su propuesta del ideal de la actividad artística como la forma de trabajo a la que debieran aproximarse el resto de trabajos.

\section{La verdadera diferencia entre el jugar y el trabajar}

En nuestra cultura occidental, el juego y el trabajo han sido habitualmente concebidos como dos esferas de acción opuestas. Curiosamente, los rasgos que se les suele atribuir se contraponen punto por punto. Si el juego es concebido como una actividad marginal, no seria, inútil, improductiva, placentera, realizada 
libremente y vinculada a la vida infantil; el trabajo suele ser visto como una actividad importante, seria, útil, productiva, esforzada, realizada por obligación y asociada a la vida adulta. Esta visión dualista era, según Dewey, el resultado de la evolución de unas determinadas convenciones culturales que, sin embargo, no hacía justicia al verdadero carácter de estas actividades. Como es sabido, toda la filosofía de Dewey fue un intento por superar el esquema de pensamiento dualista, esas falsas líneas divisorias que se han venido estableciendo entre el cuerpo y la mente, la práctica y la teoría, el artesano y el artista, o el individuo y la comunidad. A lo largo de su carrera, Dewey puso radicalmente en cuestión estos pares de opuestos y planteó en cambio una forma de pensar que, partiendo siempre de la experiencia, buscaba conectar más que separar, cooperar más que competir, resolver situaciones problemáticas concretas y cotidianas más que perderse en vagos ejercicios intelectuales.

Para Dewey, el dualismo, fuera del tipo que fuera, falseaba la realidad. Por supuesto, esos términos existían, había una materia y una forma, como poseíamos un cuerpo físico y algún tipo de entidad espiritual. El problema surgía al entenderlos como entidades separadas en vez de como partes funcionales y cambiantes de una interacción de procesos. En primer lugar, el dualismo planteaba una división y una distancia artificiales entre los términos, a lo que Dewey propuso la idea de continuidad. En segundo lugar, el dualismo tomaba sus elementos como categorías fijas y estáticas, por lo que se hallaba imposibilitado para captar una realidad que era constantemente móvil y cambiante. En tercer lugar, siempre conllevaba el predominio de una de las partes sobre la otra, es decir, implicaba una jerarquía, mientras Dewey veía las entidades como elementos funcionales que se coordinaban en unidades interactivas. En cuarto lugar, el dualismo establecía categorías mentales de opuestos construidas a espaldas de la experiencia y que después tomaba como realidades empíricas. Y en último término, el dualismo se le presentaba a Dewey como dos monismos libremente juntos y atascados, lo que lo hacía más que multiplicar por dos las dificultades del monismo. En todo proceso había siempre más de dos elementos implicados, aunque tampoco podíamos hablar a priori de una triplicidad, una tétrada o una quíntupla. Este número no importaba y, en cualquier caso, no se podía establecer de manera previa a la experiencia ${ }^{1}$.

\footnotetext{
${ }^{1}$ Ver Dewey, 1917. Al final de este artículo, sin duda influenciado por la figura de William James, Dewey declaraba que, de tener que emplear alguna etiqueta para representar su posición, la expresión "pluralismo empírico" podría ser la más apropiada.
} 
En realidad, el discurso de Dewey se movió también entre parejas de conceptos, pero la diferencia se encontraba en que, mientras el dualismo establecía esos pares como opuestos y separados por una línea divisoria, Dewey los consideraba como dos polos, dos extremos de un mismo campo, entre los cuales, por consiguiente, no había salto sino continuidad ${ }^{2}$. La manera de operar de Dewey consistió en absorber las dos partes en una unidad mayor. Puesto que esas partes se integraban siempre en un proceso más amplio, debíamos considerarlas por su carácter contextual y funcional, y no tanto por lo que podrían ser por sí mismas y de forma aislada. Esta fue la misma estrategia que siguió a la hora de abordar el significado del juego y el trabajo. Para comprender la respuesta de Dewey a esta cuestión deben tenerse en cuenta sus convicciones más características: su visión organicista y naturalista del individuo y la sociedad (bajo la influencia del evolucionismo biológico de Darwin); su sentido procesual, interactivo y plural de la realidad (inspirado por Hegel); su actitud pragmatista (en especial, respecto a la cuestión de los medios y los fines, o los procesos y los resultados); su concepto de experiencia y método experimental; su enfoque funcionalista de la psicología; o su idea de la educación como crecimiento.

Dejando a un lado las visiones y las convenciones culturales, Dewey examinó el jugar y el trabajar en el marco del crecimiento del organismo humano. En primer lugar, debíamos considerar al ser humano en su sentido biológico: como un organismo que crecía desde la sencillez a la complejidad y que se encontraba en una constante interacción con su entorno. Un organismo evolucionaba positivamente cuando mejoraba su adaptabilidad al medio, cuando el ajuste y el equilibrio entre ambos era más firme y constante. De acuerdo al enfoque evolucionista de Dewey, el organismo se hallaba en un permanente estado de acción, o lo que es lo mismo, la actividad era la esencia misma de la vida. De este modo, Dewey contradecía el planteamiento evolucionista de Herbert Spencer, el de mayor difusión en las últimas décadas del siglo XIX. En efecto, el filósofo británico partía del supuesto de que los individuos eran naturalmente reacios a cualquier tipo de actividad. Consideraba que todo organismo vivo disponía de una limitada cantidad de energía que usaba preferentemente en mantener

\footnotetext{
${ }^{2}$ La idea de continuidad, popularizada por el evolucionismo darwinista, es clave también en la respuesta de Dewey al dualismo. Darwin había mostrado que entre los seres humanos y el resto de los animales no se produce un salto ontológico, sino una continuidad gradual. Dewey extendió este principio a otros dominios y, en particular, al ámbito de la experiencia. También Charles S. Peirce defendió esta idea, a la que consideraba puramente científica y a la que denominó sinejismo. (Ver «La inmortalidad a la luz del Sinejismo», Collected Papers, 7, 565-78).
} 
su supervivencia. Su estado natural era la quietud, y necesitaba invertir un suplemento especial de energía para despertar de ese estado inerte, lo cual, implicaba un esfuerzo que en sí mismo era doloroso. El ser humano respondía igualmente a este esquema, sus esfuerzos se orientaban fundamentalmente a asegurar su supervivencia y, si acaso, en mayor grado que el resto de organismos vivos, se movilizaba para huir del dolor o buscar el placer. Así, cuando sus necesidades, su nivel de salud y sus tareas estaban satisfechas, era posible que le sobrara una parte de esa energía. Este exceso era el que empleaba en el juego, que podía entenderse entonces como la manifestación de este desbordamiento de energía superflua. De ahí que los niños, según el razonamiento de Spencer, al estar exentos de responsabilidades y deberes, jugaran más porque tenían una cantidad relativamente mayor de energía para gastar ${ }^{3}$.

Sin embargo, Dewey creía que, al sustituir el mito de la quiescencia del que erróneamente partía Spencer por la visión del ser humano como un ser fundamentalmente activo, ya no era necesario tener que buscar alguna causa especial que explicara la razón de ser del juego. La quietud, o bien venía dada por la necesidad de reposo, o bien, era un estado anómalo. En el trato continuo con su medio, el organismo se veía expuesto a estímulos y situaciones que demandaban de él sucesivas respuestas solventes. Era pura actividad y esta se desbordaba en muy diversas direcciones, por consiguiente, lo importante sería identificar las condiciones en virtud de las cuales la actividad tomaba esta o aquella forma, así como el sentido y la productividad de esa serie de acciones. Tal como Dewey había señalado en su famoso artículo «The Reflex Arc Concept in Psychology» (1896), uno de los textos fundamentales de la psicología funcionalista, el estímulo y la respuesta constituían funciones que se coordinaban dentro de una misma unidad orgánica. Así, el estímulo excitaba (y anticipaba) su propia réplica, y esta desencadenaba una serie de acciones cuyos procesos se iban inevitablemente coloreando como más o menos gratificantes y sus resultados valorando como más o menos productivos. A lo largo de su experiencia, el organismo humano iba desechando las respuestas ineficaces y quedándose con aquellas que habían cumplido su propósito. El individuo tendía a repetir estas últimas, las cuales acababan por convertirse en hábitos. Las acciones más primigenias eran espontáneas y buscaban lo agradable o lo placentero pero, a medida que el organismo iba creciendo, prefería redundar en aquellas acciones que producían algún valor, fuera material o espiritual. Los procesos se volvían entonces más complejos por el hecho de que una idea del

\footnotetext{
${ }^{3}$ Ver Spencer, 1855.
} 
resultado era sobreañadida a las actividades previas. «Si la idea del resultado funciona como un estímulo para renovar la actividad que decae, y si, además, el cumplimiento de los resultados implica una cierta selección y acuerdo de los actos que lo anteceden, tenemos un tipo de actividad suficientemente contrastada que se ha denominado trabajo» (Dewey, 1912-13, 320). De este modo, el trabajar surgiría de forma natural a partir de las actividades espontáneas. Lo que ocurre es que en el trabajo la idea de ese resultado ocasiona un curso de actividades más largo y que requiere más esfuerzo, perseverancia, control y responsabilidad. Ese compromiso con el resultado estimula el reajuste de las actividades originalmente espontáneas, obliga al individuo a tener que pensar con mayor inteligencia en la relación entre los medios y los fines. Así, como actitud mental, el trabajo «significa interés en la corporeización adecuada de un significado (una sugerencia, una finalidad, una meta) en forma objetiva a través del uso de materiales e instrumentos adecuados» (Dewey, 1910, 212). Dewey entendió el trabajo en un sentido muy amplio: toda aquella ocupación productiva que requería esfuerzo, pero que también suponía una acción inteligente que ponía en marcha el ingenio y la inventiva en aras de obtener algún valor. Visto así, ya no se trata de una actividad rutinaria que busca resultados útiles, sino de una «actividad dirigida por fines que el pensamiento establece» $(1910,213)$.

Aunque una experiencia surgía siempre como el encuentro de unas condiciones subjetivas y unas condiciones objetivas, para Dewey, la cualidad de una actividad, su acento y su coloración finales, dependía en gran parte de la actitud con la que se afrontara. Cualquier actividad era, en último término, una serie de manifestaciones externas y pasajeras de unas actitudes previas. El carácter de esa actitud (en dialéctica con las condiciones externas) determinaba el grado de implicación, el grado de disfrute o de tedio, así como el grado de calidad del resultado. De ahí que Dewey insistiera en la importancia de las actitudes ${ }^{4}$, tanto en el campo educativo (que los nińos desarrollaran una actitud investigadora y el hábito de pensar, y no tanto la memorización de una serie de conocimientos canónicos) como a la hora de afrontar un trabajo (que el trabajador pudiera sentir su labor como un fin en sí mismo). Según Dewey, que unas determinadas actividades fueran consideradas como juegos o como trabajos era el resultado de una serie de convenciones establecidas por los adultos. Una misma actividad podía abordarse como un juego o como un trabajo, la prueba de que eran convenciones acríticas lo hallábamos en el hecho de que los niños las desconocían por completo. Si nos fijamos en cómo un niño vivencia el mundo,

\footnotetext{
${ }^{4}$ Ver Dewey, 1910, 211.
} 
advertiremos que para él la realidad no es algo externo y lejano, sino que se presenta como un gran escenario que gira a su alrededor. Los objetos y los actos captan su atención si encuentra en ellos algo que concuerde con sus intereses dominantes. Si entran en su escenario, los vive como elementos de una especie de drama, y en esta representación no distingue entre una actividad lúdica y una ocupación más laboral.

El adulto es generalmente consciente de que está jugando. Siente el elemento de hacer-creer en ello, de la falta de plena realidad. Para él eso es diversión, recreación. Todo esto es debido a que el adulto considera esas actividades contrarias a los verdaderos asuntos de la vida, las cuestiones prácticas que tienen que ver con la familia, la profesión, etc.; pero el juego no puede tener tal coloración en la mente del niño porque para él no existe tal contraste. El jugar para el niño es tan serio como el trabajo para el adulto, no serio por supuesto en el sentido de destruir la espontaneidad o menoscabar la libertad, pero serio, en el sentido de que absorbe toda su atención, de ser para él en ese momento la única realidad que existe. Es un error, por lo tanto, suponer que el juego es equivalente a entretener al niño o que se opone a lo serio o incluso al esfuerzo extenuante. El niño trabaja a menudo (en el sentido de poner adelante su máxima energía) al jugar. Además, muchas de las cosas que para los adultos se han convertido en trabajo o monotonía o trabajo pesado debido a sus asociaciones con la rutina y con los fines externos, están para el niño llenas de deleite y de fascinación en el juego; como, por ejemplo, lavar los platos, el poner una mesa, etc. (Dewey, 1900, 200-201).

Además, el tono de una actitud viene dado, principalmente, por la intensidad y la dirección del «interés». El «interés» es el vehículo por excelencia a través del cual el yo interacciona con su medio. Su propia etimología, «inter-esse», «estarentre», así lo atestigua. Según Dewey, el «interés» era el instrumento que salvaba la distancia entre el individuo y los materiales y los resultados de su acción, que ocasionaba, por lo tanto, su unión orgánica ${ }^{5}$. Así, existen actividades en las que la expresión del yo es directa e inmediata. En estos casos, predomina en el individuo el interés por la propia ejecución y no por una idea ulterior. Todo juego, como toda apreciación estética, posee esta cualidad. En sus contribuciones a la Encyclopedia of Education (1912-13), en la entrada sobre el término play, Dewey afirmaba que el «juego» comprende "aquellas actividades que no son deliberadamente realizadas con el objetivo de alcanzar un resultado más allá

\footnotetext{
${ }^{5}$ Ver Dewey, 1896a, 122ss.
} 
de ellas mismas. Actividades que son placenteras en su propia ejecución, sin referencia a un propósito ulterior» (Dewey, 1912-13, 318). Por otra parte, hay actividades en las que no tenemos un verdadero interés directo, sin embargo, aun resultándonos indiferentes o desagradables en sí mismas, las practicamos porque, o bien generan parentescos y conexiones de las que no éramos conscientes, o bien esperamos obtener de ellas un producto o un valor. En estos casos, el interés es indirecto. De este modo, mientras que en el juego, el interés se enfoca en el disfrute del propio proceso; en el trabajo, el interés en la propia tarea suele ser mediato o transferido, es decir, no se ejecuta por su propio valor, sino como medio para conseguir un fin.

La diferencia entre juego y trabajo puede aclararse si se la compara con un modo más usual de enunciarla. Se dice que en la actividad del juego, el interés reside en la actividad misma, mientras que en el trabajo el interés apunta al producto o resultado en el que la actividad culmina. De ahí que el primero sea completamente libre, mientras que el último está sujeto a la finalidad que hay que conseguir. Cuando se enuncia la diferencia de esta manera tan tajante, se introduce casi siempre una separación falsa, no natural, entre proceso y producto, entre la actividad y su resultado. La verdadera distinción no hay que buscarla entre el interés por la actividad en sí misma y el interés por el resultado externo de esa actividad, sino entre el interés por la actividad tal como fluye espontáneamente en cada momento y el interés por una actividad que tiende a una culminación, a un resultado, y que, en consecuencia, posee un hilo conductor que une sus etapas sucesivas (Dewey, 1910, 213-214).

Por lo tanto, buena parte del problema se encuentra en el hecho de establecer una férrea división entre el juego y el trabajo y no tener en cuenta que tanto el uno como el otro, al ser actividades organizadas, implican un proceso y un resultado, unos determinados medios y algún tipo de finalidad ${ }^{6}$. El interés del jugador se

\footnotetext{
${ }^{6}$ Según Dewey, los medios y los fines también han sido concebidos tradicionalmente como otra forma de dualismo. Sin embargo, desde su perspectiva pragmatista, los fines en sí o absolutos constituyen una ilusión, en realidad, no es posible definir todos los fines de antemano, sino que estos deben ir surgiendo en el trato con los medios, en el propio desenvolvimiento del proceso. Los fines deben entenderse como fines-en-perspectiva que orientan y dan sentido a un determinado curso de acciones. En vez de un patrón previo y rígido, constituyen más bien un plan de acción, un proyecto falible y abierto. Y en este plan integral, los medios son cuanto menos tan importantes como el fin-en-perspectiva. Ambos se copertenecen y coimplican. Los medios «no son intrínsecamente distintos del fin. Son solo fines próximos; son el fin analizado en sus factores constituyentes» (Dewey, 1887, 318). Además, los términos
} 
enfoca en el disfrute de los medios, pero «el juego tiene un fin en el sentido de una idea directriz que da orientación a los actos sucesivos» (Dewey, 1916, 176). El jugador no realiza sus jugadas de manera fortuita o azarosa, esto desvirtuaría el juego, de algún modo, las jugadas se hilvanan de acuerdo a un cierto plan o dirección. Si bien el interés del jugador se centra en el propio proceso, el juego posee una finalidad y el jugador la tiene presente. Cuando se juega a las muñecas o a escenificar una obra de teatro, la finalidad primordial consiste en llevar adelante la representación, aunque se ejecute de forma totalmente espontánea o de acuerdo a un libreto previo, siempre hay una idea final que orienta las diferentes acciones. En el caso de los juegos de competición, la finalidad es alcanzar la victoria, pero más que un fin en sí mismo, es una idea que orienta el sentido de las acciones a ejecutar. Precisamente, cuando al jugador solo le interesa ganar a toda costa, sea por los medios que sea, el juego deja de ser juego. Para Dewey, esa actitud que solo busca la victoria convierte a esa actividad en un trabajo, puesto que el interés dominante se sitúa en ese fin, de tal modo que los medios quedan subordinados a este. Esta mutación se aprecia con claridad en el caso del deporte a nivel profesional. La actividad posee la cualidad de juego, pero la actitud de los jugadores se encuentra determinada por el objetivo para el que han sido contratados: la consecución de la victoria ${ }^{7}$.

Por su parte, en el trabajo prevalece el interés por obtener un producto, un resultado, pero para que este trabajo productivo no se convierta en un trabajo degradado, este interés debe conjugarse con un disfrute del trabajador por su labor, por un interés en recrearse en el mismo proceso productivo. Como vemos, tanto en el jugar como en el trabajar se encuentran implicados un proceso y un resultado, la diferencia entre ellos reside en la inclinación del interés, en el acento mayor o menor que se pone en los medios o en los fines. La diferencia es, por lo tanto, de grado, no de tipo ${ }^{8}$. Más aun, cuando la conciencia de juego

no son fijos y estáticos. Un medio en un determinado plan puede funcionar como fin dentro de otro plan, de igual modo que un fin-en-perspectiva no es más que la consumación de un proceso que, probablemente, servirá en un futuro como medio para otros fines. Para la respuesta de Dewey a esta cuestión, puede consultarse Dewey, 1939a. En especial, el capítulo VI. «El continuo de medios-fines».

${ }^{7}$ El sociólogo francés Roger Caillois defenderá esta misma idea en su magnífica obra Les Jeux et les Hommes. Le masque et le vertige, París, Gallimard, 1967.

${ }^{8}$ Dewey vinculó esta diferencia de grado con la que se establecía entre la racionalidad estética y la racionalidad instrumental y, a su vez, con la que se daba entre los significados «intrínsecos» y «extrínsecos». Un análisis de estas concordancias puede consultarse en la obra de Ramón del Castillo (1995). Conocimiento y acción. El giro pragmático de la filosofía. Madrid: UNED, pp. 448ss. 
y la conciencia de trabajo se apartan y se aíslan, el juego degenera en ligereza y dispersión, en un capricho azaroso e improductivo, mientras que el trabajo se convierte en pura rutina, en algo pesado y desagradable?. Para que el juego sea provechoso y tenga un sentido, para que el trabajo sea satisfactorio para el trabajador, es necesario que se produzca la confluencia de ambas actitudes ${ }^{10}$. El trabajo creativo del artista sería, según Dewey, el que mejor ejemplificaría esta integración.

El juego desempeñaba para Dewey una importante función en el crecimiento y en el equilibrio energético del individuo pero, en último término, no poseía el mismo valor que el trabajo, precisamente porque no generaba como este un verdadero producto o valor ${ }^{11}$. La mera actividad lúdica, cuando no estaba animada por algún propósito, llevaba al capricho y la dispersión (como podía ser el caso de cierta pedagogía romántica extremadamente centrada en el niño o algunos productos banales de la cultura del espectáculo). Esta actividad, en cambio, se veía enriquecida cuando conducía a algún lugar o producía algún valor. Por ello, para Dewey, el jugar cumplía un papel vital en el desarrollo del niño puesto que posibilita el aprendizaje de una gran variedad de significados. Pero para que las actividades tuvieran un sentido y fueran verdaderamente productivas, el jugar debía transformarse en trabajo (absorbiendo, en la medida de lo posible, las cualidades del juego). Dicho de otro modo, el trabajar era precedido por el jugar y crecía a partir de él. La actitud del trabajo se aprovechaba de los significados que se habían suscitado y construido en la libertad del juego. Por eso, para que el organismo humano creciera adecuadamente, para que el nińo desarrollara satisfactoriamente su personalidad, el juego debía gradualmente transformarse en trabajo en sentido pleno (labor), esto es, en actividades de cuyo proceso se

\footnotetext{
${ }^{9}$ Ver Dewey, 1910, 281ss.

${ }^{10}$ Dewey consideraba que la confluencia de ambas conciencias era fundamental para el equilibrio energético del individuo, pero también para el proceso de reflexión, y por ende, la puesta en práctica del método experimental. Un hábito inteligente de reflexión debía combinar la actitud experimentadora y la científica, los momentos de juego mental con los momentos más serios de observación e indagación. Dejar libre al pensamiento resultaba imprescindible para que se hiciera consciente de sus dogmas y prejuicios, para que imaginara las posibilidades y los límites de un problema, para que rompiera sus restricciones y alimentara, a su vez, la curiosidad por descubrir y conocer. El estado mental ideal sería el equilibrio entre ambas actitudes (Dewey, 1910, 283-284).

${ }^{11}$ Aparte de las consideraciones biologicistas, se manifiesta también en esta visión el trasfondo cultural protestante de Dewey. A diferencia de la máxima protestante de trabajar por el deber de trabajar y como forma de honrar a Dios, Dewey incorporó la necesidad del interés directo por la actividad y su consecuente disfrute.
} 
obtuviera un cierto disfrute pero que tuvieran una dirección: la producción de un valor material y/o espiritual ${ }^{12}$.

\section{EI plan de estudios de la Laboratory School}

Vamos a ver ahora cómo Dewey se sirvió de estas ideas acerca del juego y el trabajo para diseñar el currículum de la University Elementary School, una escuela de primaria adscrita a la Universidad de Chicago y que dirigió entre 1896 y $1904^{13}$. En octubre de 1895, Dewey, siendo ya director del Departamento unificado de Filosofía, Psicología y Pedagogía, convenció al presidente de la Universidad de Chicago para que destinara una modesta partida presupuestaria a la fundación de una escuela de primaria dependiente de dicho departamento. De este modo, la escuela podría cumplir un doble propósito: por una parte, servir como centro de observación y prácticas para los estudiantes de la Escuela de Maestros; y por otra, gozar de la posibilidad de implementar las ideas en las que Dewey venía trabajando en el campo educativo. Entre las diversas líneas de investigación llevadas a cabo en su Departamento, Dewey lideró un seminario, siguiendo los postulados funcionalistas, acerca del desarrollo mental del niño y cuyos descubrimientos inspiraron el currículum de la escuela ${ }^{14}$. Siendo el organismo humano un ser esencialmente activo, lo importante para Dewey residía en el carácter y la productividad de las actividades, por lo que su análisis del crecimiento se enfocó en cómo se desarrollaban el «interés» y la actitud del individuo en relación al tipo de actividades que desenvolvía. Dewey y su equipo identificaron cuatro etapas de actividad en el desarrollo mental del niño: early

\footnotetext{
${ }^{12}$ Ver Dewey, 1910, 214 ss.

${ }^{13}$ El nombre oficial del centro fue University Elementary School, sin embargo, en cuanto empezó a cobrar relevancia, fue comúnmente conocida como «Dewey School» o «Laboratory School». A Dewey le parecía muy apropiada esta última denominación, puesto que la escuela trataba de poner en práctica una serie de ideas educativas y funcionar como un laboratorio de filosofía, el mismo sentido experimental que animaba el laboratorio sociológico de la HullHouse de Jane Addams. Sin embargo, a Dewey no le gustaba demasiado usar el término por miedo a que los padres pensasen que experimentaban con sus hijos como si fueran conejillos de indias (ver Dewey, 1899, 61). Además de las propias conferencias y obras de Dewey de la época de Chicago, el libro que mejor detalla la filosofía y el día a día de la escuela es The Dewey School: The Laboratory School of the University of Chicago, 1896-1903 (D. AppeltonCentury, Nueva York, 1936), escrita por dos de sus antiguas maestras, K. C. Mayhew y A. C. Edwards.

${ }^{14}$ Acerca del contenido y los descubrimientos de este seminario, puede consultarse la serie de conferencias impartidas por Dewey bajo el título "Mental Development» en 1900 (MW.1, 192-221), así como el libro de Irving King The Psychology of Child Development (1903).
} 
infancy (hasta los dos o tres años); later infancy (hasta los siete u ocho años); childhood (hasta los trece o catorce); youth (hasta los dieciocho o veinte) ${ }^{15}$. De este modo, organizaron el currículum de la Laboratory School de acuerdo a estas etapas del desarrollo mental. Estructuraron el plan de estudios en tres períodos educativos, los cuales, descontando la etapa del bebé, se correspondían con las tres siguientes fases del desarrollo mental mencionadas: primer período: play period; segundo período: childhood; tercer período: adolescencia. Nos centraremos en la segunda y la tercera etapa, puesto que constituyeron las etapas educativas que ofertó la Laboratory School y en los que Dewey pudo implementar las ideas que hemos comentado (solo durante los dos últimos años de su existencia, la escuela impartió un curso compuesto por nińos de 12 y 13 ańos).

Dewey denominó a la primera etapa play period, lo cual no quería decir que los niños debieran dedicarse a jugar alegremente (tal como estaba de moda en muchas escuelas progresistas), sino que apuntaba al tipo de actitud preferente que mostraban los niños en ese tiempo: una manera característicamente lúdica de interaccionar con el mundo. El juego es libertad y espontaneidad, es actividad y experiencia, involucra a la imaginación, exige creatividad y suele ir acompañado de altas dosis de emoción e incertidumbre, y estos son precisamente los rasgos dominantes de los niños entre los cuatro y los ocho años de edad. Durante este período, la educación debía tener por objetivo el fomentar y fortalecer este carácter instintivo del niño. A Dewey le interesaba aprovechar esta energía desbordante que los niños ejercitaban al jugar para proponerles experiencias que después les ayudaran a transformar esa energía en actividades productivas. El juego le proporcionaba al niño una multitud de significados que aprendía a través de la actividad y sin esfuerzo. Sin embargo, tal como ya hemos subrayado, un exceso de ludismo favorecía la dispersión y el capricho. Así, el tránsito del play period al childhood period debía posibilitar que el niño transformara progresivamente su actitud lúdica natural en una actitud que supiera tratar con procedimientos complejos que buscaban un resultado valioso, en una actitud capaz de responder inteligentemente a nuevas situaciones. Esta transición se coordinaba con visión deweyana del aprendizaje: la manera más adecuada y significativa de aprender era ir de lo más familiar a lo más teórico, de lo más concreto a lo más abstracto. Dewey trató de aplicar sus ideas acerca del método experimental a la metodología pedagógica. El niño crecía física e intelectualmente a partir de la ganancia de experiencias significativas, es decir, vivencias de primera mano que le aportaban un valor. El método consistía, pues,

${ }^{15}$ Ver Dewey, 1900. 
en ofrecer primero la situación, que el niño se encontrara por sí mismo con los problemas y, a partir de ahí, investigar las posibles soluciones y estudiar las diversas materias involucradas. En consecuencia, el currículum debía proporcionar al niño situaciones activas en las que tuviera que enfrentarse por sí mismo (con la guía de los maestros) a los materiales y a los procedimientos. De este modo, para crear situaciones activas, el programa de estudios de la Laboratory School se estructuró en "ocupaciones activas» en vez de hacerlo en asignaturas al modo tradicional.

El plan de estudios establecía tres familias fundamentales de ocupaciones: el trabajo en el taller (shop-work) con madera y herramientas; el trabajo de cocina; y el trabajo con textiles, que incluía el tejido y la costura ${ }^{16}$. A estas actividades se añadían otras complementarias, a saber: el trabajo artístico (dibujo, pintura, música, canto); la práctica, o invención, de juegos (en especial, juegos colaborativos y de dramatización); el trabajo en el gimnasio (los niños dedicaban media hora al día al ejercicio físico); el cultivo de un jardín y un huerto; el trabajo en el laboratorio; así como excursiones al campo, a museos, o simplemente, paseos por el entorno de la escuela. Durante los primeros años de escolarización (play period), los niños se dedicaban fundamentalmente a la realización de estas actividades para, gradualmente, a partir del segundo grado (hacia los ocho años), ir profundizando en el estudio de las materias que esas actividades habían ido suscitando. Los niños más mayores continuaban participando de estas ocupaciones, pero empleaban más tiempo en estudiar los contenidos históricos y sociales, los científicos, y los expresivo-comunicativos, las tres ramificaciones en las que se estructuraba el currículum en el segundo período.

La base de todo crecimiento posterior es la actividad en el período anterior [...] De ahí la necesidad de que los juegos más tempranos sean de tal índole que permitan al niño crecer de manera natural y amable, y le preparen para modos de comportamiento más reflexivos y productivos. Esto significa que el juego debe transformarse imperceptiblemente en trabajo [...], y que tanto ese juego temprano como el trabajo proporcionan ejercicio en las ocupaciones socialmente provechosas. Una verdadera iniciación en ellas a través del juego no sólo significa que la persona ha adquirido, bajo condiciones de menor resistencia y mayor economía, la capacidad necesaria para elaborar juicios certeros desde el punto de vista social, sino que lo ha hecho a través

\footnotetext{
${ }^{16}$ Cfr. Dewey, 1899, 61ss.
} 
de la conexión de su propia imaginación y sus propias emociones. En otras palabras, la natural transición del juego al trabajo es el medio y el único medio de reconciliar el desarrollo de la eficiencia social con el discurrir de la vida individual (Dewey, 1912-13, 318).

Los niños iban, pues, a la escuela experimental para hacer cosas (fuera cocinar, jugar, construir una maqueta o fabricar herramientas), y a partir de la ejecución de estos actos sencillos, se iba articulando el estudio de las materias. Por ejemplo, el ejercicio de cocinar daba pie a estudiar aritmética (pesar y medir los ingredientes), química y física (observar los estados de la materia y los procesos de combustión), biología (dieta y digestión), geografía (exploración de los ambientes naturales de plantas y animales) o agricultura (los tipos de alimentos y sus formas de cultivo). De este modo, a través de la actividad, el niño aprendía una gran variedad de significados en su contexto, un background imprescindible para, durante el segundo período educativo, poder profundizar en las ideas más abstractas.

\section{La desafección del trabajador en el capitalismo industrial}

Es conocido que Dewey, aparte de un reputado académico, fue un activista incansable que se implicó de lleno en las luchas sociales y políticas de su tiempo ${ }^{17}$. En Chicago, a donde llegó en 1894, justo en el punto álgido de la Huelga Pullman, pudo apreciar de primera mano los desequilibrios provocados por la industrialización y el sistema de producción capitalista. La ciudad había celebrado la Exposición Universal en 1893, un acontecimiento que le permitió dar a conocer al mundo su pujanza y que supuso la llegada de miles de personas atraídas por las oportunidades de empleo. Sin embargo, el 27 de junio de 1893 el mercado de valores de Nueva York se desplomó, terminando con el ciclo comercial de los «Gilded Years» y desencadenando una profunda depresión. Al terminar la exposición, la crisis llevó a la quiebra, solo en el área de Chicago, a seiscientos bancos y 15.000 empresas, dejando a más de 300.000 personas sin trabajo. Dewey llegó a la ciudad en aquellos convulsos meses y desde el

\footnotetext{
${ }^{17}$ Acerca de las actividades políticas de Dewey y el contexto social de Estados Unidos en las décadas de los años 20 y 30, son excelentes y muy ilustrativos los textos de Ramón del Castillo: (2003). "El amigo americano", Introducción a Dewey, J., Viejo y nuevo individualismo, Barcelona: Paidós; (2004). «Érase una vez en América: John Dewey y la crisis de la democracia», estudio preliminar, bibliografía, revisión y notas a Dewey, J., La opinión pública y sus problemas, Madrid: Morata, pp. 11-56.
} 
primer momento se solidarizó con las reivindicaciones de los trabajadores. Allí vio cómo el creciente desempleo condenaba a muchas familias a la pobreza y la marginalidad. Chicago comenzaba a parecerse demasiado al Manchester de la revolución industrial que tan bien había retratado Engels.

Aunque el sistema capitalista presumía de optimizar sus recursos y producir una riqueza material que no tenía parangón en la historia, Dewey creía que se trataba de un orden social preocupantemente ineficaz en cuanto al aprovechamiento de la energía humana. La distribución y la producción se organizaban en función del beneficio pecuniario, desperdiciando así una cantidad incalculable de capacidades y recursos humanos. La causa de este despilfarro era el desempleo, a juicio de Dewey, la más grave de las deficiencias del sistema y un síntoma evidente de incivilización. El desempleo degradaba las condiciones de vida de los individuos, y con ello, se alimentaban la amargura, la hostilidad, la corrosión del carácter y el debilitamiento de las convicciones morales ${ }^{18}$. Si el individuo conseguía emplearse en alguna fábrica, apenas alcanzaba a mejorar mínimamente su vida a cambio de un trabajo que difícilmente le reportaría alguna satisfacción. La organización fabril, al ir desplazando a los talleres, había transformado por completo la forma de trabajar. Con la mecanización de los procesos de producción (la cadena de montaje o el posterior fordismo) y la división y la especialización de las tareas, el trabajador se convertía en un gestor de maquinaria que solo realizaba una labor muy parcial que no requería de una competencia cualificada. En este contexto, el trabajador industrial se veía privado de su creatividad, del tratamiento directo con los materiales, del valor y la honra de poseer una capacidad especial, de la propiedad y el control tanto de los procedimientos como de los fines y, en definitiva, de la posibilidad de vivir su trabajo como una experiencia satisfactoria y productiva. Dewey, aun compartiendo la reivindicación de los trabajadores por la mejora de sus condiciones laborales (jornadas de ocho horas, salarios dignos, seguro médico o el derecho a un subsidio por desempleo), creía que la consecución de estos derechos no evitaría que los trabajadores continuaran sintiendo su trabajo como algo ajeno y extraño, como un simple medio para obtener un salario y no como un fin en sí mismo.

Tal como señalamos anteriormente, Dewey comprendió el trabajo en un sentido muy amplio: toda aquella actividad productiva que requería esfuerzo e ingenio por nuestra parte y que constituía un proceso complejo en el que la

${ }^{18}$ Ver Dewey, 1918. 
idea de un resultado guiaba los pasos a seguir. Esta actividad se convertía en una auténtica experiencia para el individuo cuando alcanzaba una concreción refinada del fin que se proponía, pero para este éxito era imprescindible prestar atención y saber manejar los procedimientos, en los términos deweyanos, mostrar un interés especial por el propio proceso. Por lo tanto, una experiencia auténtica exigía la confluencia del interés por el proceso (actitud lúdica) y el interés por el resultado (la actitud hacia el trabajo). El trabajo laboral, no siendo más que un subconjunto del total de actividades productivas que realizábamos, debiera también responder a esta confluencia para que no se degradara en una vivencia carente de significado. Para que el trabajador pudiera experimentar su trabajo como un espacio de realización personal, debía mostrar un interés en los fines y los resultados de la actividad, sentir que participaba de ellos, pero a su vez, ese compromiso debía conjugarse con un disfrute del trabajador con su labor, con un interés en recrearse en el mismo proceso productivo. Cuanta más habilidad y creatividad pudiera aportar por sí mismo al procedimiento, mayor sería el grado de satisfacción que obtendría y mayor sería la calidad del resultado $^{19}$. Al ser la actitud del artista o el artesano (no habría para Dewey diferencias sustanciales entre ellos) la que mejor ejemplificaría la confluencia de la conciencia lúdica y la conciencia del trabajo, al ser su actividad artística la que mejor expresaría la integración de los medios y los fines, este modo de trabajo constituiría el ideal ${ }^{20}$ al que debieran aproximarse el resto de trabajos ${ }^{21}$. Para materializar este ideal, en parte inspirado por el movimiento Arts and

\footnotetext{
${ }^{19}$ Aunque Dewey reconoció no haber estudiado seriamente a Marx, y aunque rechazó la filosofía social marxista, por ser totalitaria, monolítica, dogmática y atentar contra las libertades individuales (Ver Dewey, 1939b), su crítica fue similar a la que Marx planteó en sus Manuscritos a partir de la noción de «trabajo alienado». Ambos creían que la economía debía ser un espacio de realización de las capacidades humanas y ambos comprendían el trabajo como una forma más de actividad productiva. No obstante, para Marx, el espacio que ocupa lo económico debía en último término reducirse al mínimo (algo que solo se lograría en la sociedad comunista), puesto que debemos disfrutar la vida por sí misma y no tener que emplear la mayor parte de nuestro tiempo en los medios para sostenerla. Frente al «trabajo enajenado» al que se veía forzado el trabajador industrial (desposeído de sus medios y fines, extraño respecto al procedimiento, el producto y su propio ser genérico), para Marx (como para Dewey), el auténtico trabajo sería aquel que el trabajador realice libremente, como un fin en sí mismo y no como un simple medio, aquel en el que pueda desarrollar sus potencialidades (Marx, [1844] 2013).

${ }^{20}$ Debe entenderse ideal en el sentido característico de la tradición pragmatista: como un finen-perspectiva que orienta nuestras acciones, surgido de nuestro trato con el mundo, no infalible y revisable.

${ }^{21}$ Dewey abordó esta cuestión en distintos lugares de su obra, fundamentalmente en How We Think (1910), Democracy and Education (1916), y Art as Experience (1934).
} 
Crafts de William Morris y John Ruskin, en parte heredero del republicanismo de Jefferson, Dewey abogó por instituir una autonomía industrial, es decir, el control por parte de los trabajadores de las condiciones y los procedimientos de su trabajo. Planteó un liberalismo social, que incluía la reclamación de que el trabajo debía poseer un significado y un valor para el trabajador; la instauración de un Consejo Económico de ámbito nacional, formado por representantes de los trabajadores, empresarios y funcionarios del gobierno que planificara la economía y la industria en función del interés general; o también, la creación de un órgano directivo en las empresas, compuesto por trabajadores y directivos, encargado de planear los medios y los fines de la producción ${ }^{22}$.

La nueva sociedad capitalista también había instaurado una cultura y una industria del ocio asociada al consumo y el entretenimiento, las cuales tuvieron en la Exposición Universal de Chicago de 1893 uno de sus momentos fundacionales ${ }^{23}$. Una vez que se extendió en Estados Unidos la jornada de ocho horas, la gente comenzó a disponer de un cierto tiempo de ocio. Al individuo se le presentaron entonces una amplísima variedad de actividades lúdicas y espectáculos a los que entregarse al terminar su jornada laboral ${ }^{24}$. Dewey creía que estas actividades podían aportarle al individuo una oportunidad para socializar, para divertirse o relajarse. Sin embargo, a ojos de Dewey, esta cultura se estaba extendiendo de forma preocupante, al tiempo que se volvía más y más banal, contribuyendo a distraer a la gente de los asuntos políticos y a apartarla de la participación activa en lo público. Peor aún que esta desafección era el

\footnotetext{
${ }^{22}$ Acerca del liberalismo social de Dewey y el programa de medidas que propuso, su artículo «Internal Social Reorganization after the War» (1918) es muy esclarecedor. Por supuesto, también sus obras de pensamiento político más importantes de los años 20 y 30: The Public and its Problems (1927), Individualism, Old and New (1930), Liberalism and Social Action (1935), y Freedom and Culture (1939).

${ }^{23}$ Sobre este hito, puede consultarse la estupenda obra de Marinas, J.-M., y Santamarina, C. (2015). El bazar americano en las exposiciones universales, Madrid: Biblioteca Nueva, pp.127168.

${ }^{24}$ El sociólogo David Riesman (1909-2002) investigó de forma soberbia el tránsito de la sociedad industrial a la sociedad de consumo en los Estados Unidos, una evolución que se inició en la conocida como era progresista (entre la crisis de 1893 y la I Guerra Mundial) y que se consumó a partir de la década de los años 20. Comparó el significado del trabajo y el uso que hacían del tiempo de ocio el estadounidense típico de la primera sociedad (el individuo dirigido internamente) con el estadounidense típico de la segunda (el individuo dirigido por los otros). Ver Riesman, D. (con la colaboración de Nathan Glazer y Revel Denney) (1950). The Lonely Crowd. A Study of the Changing American Character, Yale University Press. Traducción española: (1981). La muchedumbre solitaria. Un estudio sobre la transformación del carácter norteamericano, Barcelona: Paidós.
} 
caso de muchos individuos que, al no obtener de su trabajo más que pesadez y desmotivación, buscaban de forma compulsiva actividades que les ocasionaran un placer intenso e inmediato. El problema, psicológico y social, era que el uso del ocio y de las actividades lúdicas se convertía en una forma de evasión. El alcohol, las drogas, las apuestas o el shopping constituían fenómenos demasiado extendidos en la sociedad estadounidense que respondían a esa demanda de escape. El capitalismo empujaba a mucha gente a la pobreza y, además, según Dewey, estaba alimentando una cultura consumista y hedonista entre la gente acomodada. Una cultura que fomentaba individuos inmaduros y caprichosos $y$, en consecuencia, relaciones sociales superficiales ${ }^{25}$. En estas circunstancias, el individuo veía empobrecida su vida, la cual se desdoblaba en dos espacios de acción aislados y separados. Por un lado, un trabajo pesado y desagradable; por otro, un ocio basado en el consumo y en el entretenimiento, sino en una forma de evasión. Frente a esta división y este aislamiento de la actitud lúdica y la actitud hacia el trabajo, Dewey defendió la necesidad de hacer confluir ambas actitudes. Solo así podría el individuo sentir su trabajo como una experiencia plena, mientras aprovechaba su tiempo de ocio para recuperar energías y para vivir experiencias gratificantes que le proporcionaran algún tipo de valor.

\section{Conclusiones}

Siguiendo a Dewey, esperamos haber mostrado que la visión dualista dominante en nuestras sociedades occidentales acerca del juego y el trabajo es el resultado de unas determinadas convenciones culturales. Una prueba de que esta perspectiva es el fruto de unas convenciones acríticas establecidas por los adultos es el hecho de que los niños no poseen noción alguna de esta distinción. Desde el punto de vista psicológico, la actitud lúdica sitúa el acento de sus intereses en el propio procedimiento, mientras la actitud hacia el trabajo pone su acento en el producto, pero ambos intereses se entremezclan, la verdadera diferencia reside en el énfasis en uno u otro. Por lo tanto, entre ambas actitudes se establece una continuidad y no necesariamente un corte o salto cualitativo, su diferencia no es de tipo sino de grado. Precisamente, cuando ambas conciencias se apartan y se aíslan, el juego degenera en ligereza, en un capricho azaroso e improductivo, mientras el trabajo se convierte en pura rutina, en algo pesado y desagradable. Siendo tan perjudicial esta división, Dewey señaló entonces que la única vía para vivir experiencias auténticas pasaba por hacer confluir las dos

${ }^{25}$ Ver Dewey, 1927, 132ss. 
actitudes. El trabajo artístico representaba el mejor ejemplo de esa confluencia, de ahí que, para Dewey, tanto la actividad del nińo y la tarea del maestro en el campo educativo, como el trabajo del trabajador en las nuevas condiciones de la economía capitalista, debían estar animadas por el mismo esfuerzo, la misma pasión y la misma creatividad que pone un artista en la realización de su obra.

En mi opinión, la actualidad del planteamiento educativo de Dewey está fuera de toda duda. En un momento en el que los juegos han llegado a convertirse en una moda en el plan de estudios de muchas de nuestras escuelas, Dewey nos advierte del peligro de un exceso de ludismo en el aprendizaje del niño y cómo la actitud lúdica, característica de los niños entre los cuatro y los ocho años, debe ir transformándose en una actitud hacia el trabajo. La relevancia de la pedagogía deweyana se manifiesta con claridad en el sistema educativo de Finlandia, cuyas escuelas públicas funcionan desde hace tiempo con la metodología de proyectos. No es casualidad que este país haya copado los primeros puestos del Informe Pisa desde que este empezó a elaborarse en el año 2000.

Respecto a la crítica de Dewey a la degradación de las condiciones de vida y del trabajo laboral en el nuevo sistema capitalista, lo cierto es que su respuesta no fue muy distinta a la que plantearon los anarquistas, socialistas, comunistas o progresistas de su época. Todos ellos, aun con sus diferencias programáticas, reivindicaron una solución a la conocida como "cuestión social», esto es, la pobreza y las graves desigualdades provocadas por la industrialización. Creo que el acierto y la originalidad de Dewey en este asunto reside en que, aparte de enseñarnos cómo se debe trabajar con las ideas abstractas para arrojar luz sobre distintos campos de experiencia, sitúa el foco sobre la relación entre la actitud lúdica y la actitud hacia el trabajo, mostrando cómo esta se encuentra en la raíz de problemáticas de índole psicológica y social (estas últimas, además, íntimamente conectadas). Vista desde hoy en día, su propuesta del trabajo del artista como ideal al que debieran acercarse el resto de trabajos puede parecer demasiado optimista, pero si aspiramos a vivir experiencias significativas, ello pasará por hacer confluir la libertad y la creatividad de lo lúdico con el esfuerzo y el rigor del trabajo. 


\section{Bibliografía}

Caillois, R. (1967). Les Jeux et les Hommes. Le masque et le vertige, París: Gallimard. Traducción española: (1994). Los juegos y los hombres. La máscara y el vértigo, México D.F.: Fondo de Cultura Económica.

Del castillo, R. (1995). Conocimiento y acción. El giro pragmático de la filosofía, Madrid: UNED.

- (2003). «El amigo americano», Introducción a Dewey, J., Viejo y nuevo individualismo, Barcelona: Paidós.

— (2004). «Érase una vez en América: John Dewey y la crisis de la democracia», estudio preliminar, bibliografía, revisión y notas a Dewey, J., La opinión pública y sus problemas, Madrid: Morata, pp. 11-56.

- (2013). «Una grammatica dell'esperienza. John Dewey e le lotte culturali», Il pensiero di John Dewey tra psicologia, filosofia, pedagogia. Prospettive interdisciplinari, E. Frauenfelder, M. Striano y S. Oliverio (eds.), Fridericiana Editrice Universitaria, Napoli, pp. 213-251 (incluye réplicas de R. Calcaterra y G. Mari. Traducción al italiano de S. Oliverio).

— (2014). «Pobres diablos: José Gaos, John Dewey y la metafísica made in USA», primera parte en Diánoia, volumen LIX, número 72 (mayo 2014), pp. 131-153; segunda parte en Diánoia, volumen LIX, número 73 (noviembre 2014), pp. 113-133.

- (2017). «Libertad en condiciones. A vueltas con Dewey y Lippmann», El debate Lippmann-Dewey y la democracia deliberativa, Aznar, H. (ed.), Tecnos, Madrid [en pren$\mathrm{sa}$.

Dewey, J. (1967-1991). The Collected Works of John Dewey. The Early Works (18821898). The Middle Works (1899-1924). The Later Works (1925-1953), Carbondale: Southern Illinois University Press. Empleamos las abreviaturas EW para The Early Works, MW para The Middle Works, y LW para The Later Works, seguidas del volumen y los números de página correspondientes.

- (1887). Psychology, New York: Harper and Brothers. EW 2: 1-366.

- (1896a). «Interest in Relation to Training of the Will», Second Supplement to the First Yearbook of the National Herbart Society, 209-46. Revisado y reimpreso en University of Chicago Press, Chicago, 1899. EW 5: 113-146.

- (1896b). "The Reflex Arc Concept in Psychology», Psychological Review III, 357-370. EW 5: 96-109. Traducción española en Faerna, Á. (ed.) (2010). «El concepto de arco reflejo en psicología». La miseria de la epistemología: Ensayos de pragmatismo. Madrid: Biblioteca Nueva.

- (1899). The School and Society, Chicago: University of Chicago Press. MW 1: 1-109. 
— (1900). «Mental Development», Chicago: University of Chicago (mimeografiado). MW 1: 192-221.

- (1910). How We Think, Lexington (MA): D. C. Health and Company. MW 6: 177356. Traducción española: (2010). Cómo pensamos. La relación entre pensamiento reflexivo y proceso educativo. Barcelona: Paidós.

- (1912-13). «Contributions to A Cyclopedia of Education», A Cyclopedia of Education Vol. 3, 4, and 5, New York: Macmillan Co. MW 7: 318-323.

- (1916). Democracy and Education. An Introduction to the Philosophy of Education, New York: The Macmillan Company. MW 9: 1-370. Traducción española: (2004). Democracia y educación. Madrid: Morata.

- (1917). "Duality and Dualism», Journal of Philosophy, Psychology and Scientific Methods 14, 491-493. MW 10: 64-66.

- (1918). «Internal Social Reorganization after the War», Journal of Race Development 8, 385-400. MW 11: 73-86.

- (1927). The Public and its Problems. An Essay in Political Inquiry, New York: Henry Holt and Co. LW 2: 235-431. Traducción española: (2004). La opinión pública y sus problemas, Madrid: Morata.

- (1930). Individualism, Old and New, New York: Minton, Balch and Co. (artículos previamente publicados en The New Republic). LW 5: 41-123. Traducción española: (2003). Viejo y nuevo individualismo, Barcelona: Paidós.

- (1934). Art as Experience, New York: Minton, Balch and Co. LW 10: 1-456. Traducción española: (2008). El arte como experiencia, Barcelona: Paidós.

- (1935). Liberalism and Social Action, New York: G. P. Putnam's Sons. LW 11: 1-65. Traducción española: (1996). Liberalismo y acción social y otros ensayos, Valencia: Edicions Alfons el Magnànim.

- (1939a). Theory of Valuation, Chicago: University of Chicago Press. LW 13: 189-251. Traducción española: Teoría de la valoración, Madrid: Siruela, 2008.

- (1939b). Freedom and Culture, New York: Capricorn Books. LW 13: 63-188. Traducción española: (1965). Libertad y cultura, México D. F.: Uteha.

KInG, I. (1903). The Psychology of Child Development, Chicago: The University of Chicago Press.

Marinas, J.-M. y Santamarina, C. (2015). El bazar americano en las exposiciones universales, Madrid: Biblioteca Nueva.

Marx, K. ([1844] 2013). Manuscritos de economía y filosofia, Madrid: Alianza.

Mayhew, K. C., y Edwards, A. C. (1936). Dewey School: The Laboratory School of the University of Chicago, 1896-1903, Nueva York: D. Appelton-Century. 
Peirce, Ch. S. (1931-1958). Collected Papers Vol. 1-8, Cambridge: Harvard University Press.

Riesman, D. (con la colaboración de Nathan Glazer y Revel Denney) (1950). The Lonely Crowd. A Study of the Changing American Character, Yale University Press. Traducción española: (1981). La muchedumbre solitaria. Un estudio sobre la transformación del carácter norteamericano, Barcelona: Paidós.

Spencer, H. (1855). The Principles of Psychology, London: Longman, Brown, Green and Longmans.

Recibido: 9/06/2016

Aceptado: 11/10/2017

\section{(c) $(1) \Theta$}

ENDOXA está bajo una licencia de Creative Commons Reconocimiento-NoComercial-SinObraDerivada 4.0 Internacional 